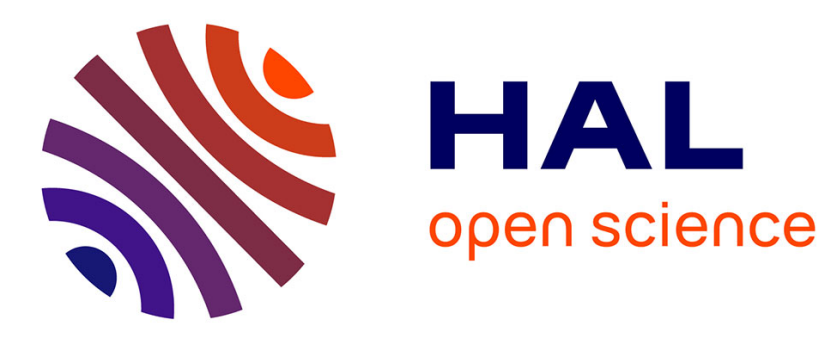

\title{
Monotone AC-Tree Automata
}

Hitoshi Ohsaki, Jean-Marc Talbot, Sophie Tison, Yves Roos

\section{To cite this version:}

Hitoshi Ohsaki, Jean-Marc Talbot, Sophie Tison, Yves Roos. Monotone AC-Tree Automata. 12th International Conference on Logic for Programming Artificial Intelligence and Reasoning, 2005, Montego Bay, Jamaica. pp.337-351. inria-00536693

\section{HAL Id: inria-00536693 https://hal.inria.fr/inria-00536693}

Submitted on 16 Nov 2010

HAL is a multi-disciplinary open access archive for the deposit and dissemination of scientific research documents, whether they are published or not. The documents may come from teaching and research institutions in France or abroad, or from public or private research centers.
L'archive ouverte pluridisciplinaire HAL, est destinée au dépôt et à la diffusion de documents scientifiques de niveau recherche, publiés ou non, émanant des établissements d'enseignement et de recherche français ou étrangers, des laboratoires publics ou privés. 


\title{
Monotone AC-Tree Automata
}

\author{
Hitoshi Ohsaki ${ }^{1}$, Jean-Marc Talbot ${ }^{2}$, Sophie Tison ${ }^{2}$ and Yves Roos ${ }^{2}$ \\ ${ }^{1}$ National Institute of Advanced Industrial Science and Technology \\ and \\ PRESTO, Japan Science and Technology Agency \\ ohsaki@ni.aist.go.jp \\ ${ }^{2}$ Laboratoire d'Informatique Fondamentale de Lille \\ Université des Sciences et Technologies de Lille, France \\ \{talbot,tison,yroos\}elifl.fr
}

\begin{abstract}
We consider several questions about monotone AC-tree automata, a class of equational tree automata whose transition rules correspond to rules in Kuroda normal form of context-sensitive grammars. Whereas it has been proved that this class has a decision procedure to determine if, given a monotone AC-tree automaton, it accepts no terms, other important decidability or complexity results have not been well-investigated yet. In the paper, we prove that the membership problem for monotone AC-tree automata is PSPACE-complete. We then study the expressiveness of monotone AC-tree automata: precisely, we prove that the family of AC-regular tree languages is strictly subsumed in that of AC-monotone tree languages. The proof technique used in obtaining the above result yields the answers to two different questions, specifically that the family of monotone AC-tree languages is not closed under complementation, and that the inclusion problem for monotone AC-tree automata is undecidable.
\end{abstract}

Keywords : equational tree automata, closure properties, decidability, complexity.

\section{Introduction}

Tree automata [5] have been applied successfully in many areas of computer science, such as protocol verification $[1,12]$, type inference $[7,11]$, checking the sufficient completeness of algebraic specifications $[3,16]$, and checking the consistency of semistructured documents [17]. This widespread use is due to good closure properties of tree automata, such as the (effective) closedness under Boolean operations and rewrite descendant computation, as well as efficient decision procedures. However, the standard framework of tree automata is not powerful when some algebraic laws such as associativity and commutativity have to be taken into account. In particular, it is known that the regularity of tree languages is not preserved for the congruence closure with respect to an equational theory. To overcome this problem, Ohsaki [23] in 2001 and Goubault-Larrecq and Verma [14] in 2002 independently proposed extensions of tree automata. Their ideas in new frameworks are to combine tree automata with equational theories, and each of their studies considers by coincidence the case in particular where some of the function symbols have associative (A), commutative (C), and/or some other 
equational properties like the identity (I) and nilpotent (U) axioms. The notion of accepted languages may differ for these two approaches, however, they coincide in the regular case for any combination of the axioms A, C, I and U.

The AC case is of particular interest since this kind of automata which are able to deal with AC symbols are closely related to tree automata with arithmetical constraints, such as multitree automata [21] and Presburger tree automata [29]. Further discussion on this relationship can be found in our recent paper [2]. It has been shown that for AC-tree automata good properties of "classical" tree automata remain: the membership and emptiness are decidable and the closure of automata by Boolean operations can be computed [23,30,31].

Motivated by cryptographic protocol verification, Goubault-Larrecq and Verma proposed to extend AC-tree automata by considering two-way and/or alternating computations [14]. They proved on one hand that two-way AC-tree automata are not more powerful than (one-way) AC-tree automata. On the other hand, the alternation strictly increases the expressiveness of $\mathrm{AC}$-tree automata while the emptiness problem is undecidable.

Inspired by commutative grammars [13,27] (alternatively, called multiset grammars [19]) Ohsaki proposed another extension of AC-tree automata [23], called monotone AC-tree automata; he proved that both emptiness and membership remain decidable for monotone AC-tree automata and that the languages defined by these automata are closed under union and intersection [23,25]. Furthermore, Ohsaki and Takai develop the automated system, called ACTAS, manipulating AC-tree automata computation by using the exact and approximation algorithms [26].

In this paper, we further investigate monotone AC-tree automata. First, we prove that the membership problem of deciding, "given a term $t$ and an automaton $\mathcal{A} / \mathrm{AC}$, whether $t$ belongs to the language defined by $\mathcal{A} / \mathrm{AC}$ " is PSPACE-complete: we give a nondeterministic algorithm running in polynomial space with respect to the size of the input tree and automaton. For the lower bound, we reduce the validity problem of quantified Boolean formulas to the membership problem. Then we show that the class of monotone AC-tree automata is strictly wider than the class of regular AC-tree automata by exhibiting a tree language accepted by a monotone AC-tree automaton but that cannot be defined by any regular $\mathrm{AC}$-tree automaton. Following the same ideas, we prove that the family of AC-monotone tree languages is not closed under complement while this class is closed under union and intersection. Finally, using similar techniques, we show that the inclusion problem for monotone AC-tree automata is not decidable.

The paper is organized as follows. Definitions and terminologies concerning equational tree language theory are introduced in Section 2. The closure properties and the decidability of equational tree automata are also summarized. In Section 3, we discuss the complexity of the membership problem for monotone AC-tree automata, proving that the problem is PSPACE-complete. Section 4 is devoted to the study of the relative expressiveness of AC-tree automata. Using the proof technique introduced in the previous section, we show in Section 5 that AC-monotone tree languages are not closed under complementation. Section 6 contains the proof for the undecidability of the inclusion problem. Finally, we conclude by summarizing the results obtained in the paper that give us the solutions to open questions in [6]. 


\section{Preliminaries}

A signature is a finite set $\mathcal{F}$ of function symbols together with natural numbers $n$. A natural number $n$ associated with $f$, denoted by arity $(f)=n$, is the arity of $f$. Function symbols of arity 0 are called constants. We assume the existence of countably infinite set $\mathcal{V}$ of variables. The set $\mathcal{T}(\mathcal{F}, \mathcal{V})$ of terms over $\mathcal{F}$ with $\mathcal{V}$ is inductively defined as follows: $\mathcal{V} \subseteq \mathcal{T}(\mathcal{F}, \mathcal{V}) ; f\left(t_{1}, \ldots, t_{n}\right) \in \mathcal{T}(\mathcal{F}, \mathcal{V})$ if $\operatorname{arity}(f)=n$ and $t_{i} \in \mathcal{T}(\mathcal{F}, \mathcal{V})$ for all $1 \leqslant i \leqslant n$. Elements in the set $\mathcal{T}(\mathcal{F}, \varnothing)$ are called ground terms. In the paper, we write $\mathcal{T}(\mathcal{F})$ for $\mathcal{T}(\mathcal{F}, \varnothing)$.

Let $\square$ be a fresh constant, named a hole. Elements in the set $\mathcal{T}(\mathcal{F} \cup\{\square\}, \mathcal{V})$ of terms, denoted by $\mathcal{C}(\mathcal{F}, \mathcal{V})$, are contexts. The empty context is the hole $\square$. If $C$ is a context with $n$ holes and $t_{1}, \ldots, t_{n}$ are terms, then $C\left[t_{1}, \ldots, t_{n}\right]$ represents the term obtained from $C$ by replacing the holes from left to right by $t_{1}, \ldots, t_{n}$ in $\mathcal{F}(\mathcal{F}, \mathcal{V})$. Terms $t_{1}, \ldots, t_{n}$ are subterms of $C\left[t_{1}, \ldots, t_{n}\right]$.

A tree automaton (TA for short) $\mathcal{A}$ is a 4-tuple $\left(\mathcal{F}, \mathcal{Q}, \mathcal{Q}_{\text {fin }}, \Delta\right)$, whose components are the signature $\mathcal{F}$, a finite set $\mathcal{Q}$ of states such that $\mathcal{F} \cap \mathcal{Q}=\varnothing$, a subset $\mathcal{Q}_{\text {fin }}$ of $\mathcal{Q}$ consisting of final states, and a finite set $\Delta$ of transition rules whose shapes are in one of the following types:

$$
\text { (TYPE 1) } f\left(p_{1}, \ldots, p_{n}\right) \rightarrow q \quad \text { (TYPE 2) } \quad f\left(p_{1}, \ldots, p_{n}\right) \rightarrow f\left(q_{1}, \ldots, q_{n}\right)
$$

for some $f \in \mathcal{F}$ with $\operatorname{arity}(f)=n$ and $p_{1}, \ldots, p_{n}, q, q_{1}, \ldots, q_{n} \in \mathcal{Q}$.

An equational system (ES for short) $\mathcal{E}$ is a set of equations $s=t$, where $s, t$ are terms over the signature $\mathcal{F}$ with the set $\mathcal{V}$ of variables. For two terms $s, t$, we write $s={ }_{\mathcal{E}} t$ whenever $s, t$ are equivalent modulo the equational system $\mathcal{E}$, i.e. $s, t$ are the elements in the same equivalence class of the quotient term model $\mathcal{T}(\mathcal{F}, \mathcal{V}) /=\mathcal{E}$. The associativity and commutativity axioms for a binary function symbol $f$ in $\mathcal{F}$ are the equations

$$
f(f(x, y), z)=f(x, f(y, z)) \quad f(x, y)=f(y, x),
$$

respectively, where $x, y, z$ are variables in $\mathcal{V}$. In the paper, we write $\mathcal{F}_{\mathrm{A}}$ for the set of binary function symbols with associativity laws only, and $\mathcal{F}_{\mathrm{AC}}$ for the set of binary symbols equipped with both associativity and commutativity. The ES A consists of the associativity axioms for each $f \in \mathcal{F}_{\mathrm{A}}$, and $\mathrm{AC}$ is the ES consisting of the associativity and commutativity axioms for each $f \in \mathcal{F}_{\mathrm{AC}}$.

An equational tree automaton (ETA for short) $\mathcal{A} / \mathcal{E}$ is a pair of a TA $\mathcal{A}$ and an ES $\mathcal{E}$ over the same signature $\mathcal{F}$. An ETA $\mathcal{A} / \mathcal{E}$ is called

- regular if it has only rules of TYPE 1 ,

- monotone if it has rules of TYPE 1 and/or TYPE 2.

We say $\mathcal{A} / \mathcal{E}$ is a $\mathrm{AC}$-TA $(\mathrm{A}-\mathrm{TA})$ if $\mathcal{E}=\mathrm{AC}$ (resp. $\mathcal{E}=\mathrm{A}$ ). Besides, in the following discussion, we suppose $\mathcal{F}_{\mathrm{A}}=\varnothing$ when considering $\mathcal{A} / \mathrm{AC}$; likewise, $\mathcal{F}_{\mathrm{AC}}=\varnothing$ for $\mathcal{A} / \mathrm{A}$. The readers are recommended to consult [23] for a more detailed presentation.

We write $s \rightarrow \mathcal{A} / \mathcal{E} t$ if there exist $s^{\prime}, t^{\prime}$ such that $s={ }_{\mathcal{E}} s^{\prime}, s^{\prime}=C[l], t={ }_{\mathcal{E}} t^{\prime}$ and $t^{\prime}=C[r]$ for some transition rule $l \rightarrow r \in \Delta$ and context $C \in \mathcal{C}(\mathcal{F} \cup \mathcal{Q})$. This relation $\rightarrow \mathcal{A} / \mathcal{E}$ on $\mathcal{T}(\mathcal{F} \cup \mathcal{Q})$ is called a move relation of $\mathcal{A} / \mathcal{E}$. The transitive closure and reflexive-transitive closure of $\rightarrow \mathcal{A} / \mathcal{E}$ are denoted by $\rightarrow_{\mathcal{A} / \mathcal{E}}^{+}$and $\rightarrow_{\mathcal{A} / \mathcal{E}}^{*}$, respectively. For an ETA $\mathcal{A} / \mathcal{E}$ with $\mathcal{E}=\varnothing$, we simply write $\rightarrow \mathcal{A}, \rightarrow_{\mathcal{A}}^{+}$and $\rightarrow{ }_{\mathcal{A}}^{*}$, instead. 


\begin{tabular}{|c|c|c|c|}
\hline & $\begin{array}{c}\text { regular } \\
\text { AC-TA }\end{array}$ & $\begin{array}{c}\text { monotone } \\
\text { A-TA }\end{array}$ & $\begin{array}{c}\text { monotone } \\
\text { AC-TA }\end{array}$ \\
\hline closure underunion, intersection & Yes [4] & Yes & Yes \\
\hline closure under complement & Yes $[4]$ & Yes & $?$ \\
\hline decidability of emptiness & Linear & No & Yes \\
\hline decidability of membership & NP-complete & PSPACE-complete & $?$ \\
\hline decidability of inclusion & Yes & No & $?$ \\
\hline
\end{tabular}

Fig. 1. Some closure properties and decidability results

A term $t$ is accepted by $\mathcal{A} / \mathcal{E}$ if $t \in \mathcal{T}(\mathcal{F})$ and $t \rightarrow_{\mathcal{A} / \mathcal{E}}^{*} q$ for some $q \in \mathcal{Q}_{\text {fin }}$. Elements in the set $\mathcal{L}(\mathcal{A} / \mathcal{E})$ are ground terms accepted by $\mathcal{A} / \mathcal{E}$. A tree language $L$ over $\mathcal{F}$ is a subset of $\mathcal{T}(\mathcal{F})$. A tree language $L$ is $\mathcal{E}$-regular $(\mathcal{E}$-monotone) if there exists some regular (resp. monotone) $\mathcal{E}$-tree automaton $\mathcal{A} / \mathcal{E}$ such that $L=\mathcal{L}(\mathcal{A} / \mathcal{E})$. If $L$ is $\mathcal{E}$ regular with $\mathcal{E}=\varnothing$, we say $L$ is regular. Likewise, we say $L$ is monotone if $L$ is $\varnothing$-monotone.

Let op be an $n$-ary mapping from $\wp(\mathcal{T}(\mathcal{F}))^{n} \mapsto \wp(\mathcal{T}(\mathcal{F}))$. The family of $\mathcal{E}$-regular (resp. $\mathcal{E}$-monotone) languages is closed under op if whenever $L_{1}, \ldots, L_{n}$ are $\mathcal{E}$-regular (resp. $\mathcal{E}$-monotone) languages then so is op $\left(L_{1}, \ldots, L_{n}\right)$. We say that the family of $\mathcal{E}$ regular (resp. $\mathcal{E}$-monotone) languages is effectively closed under op if there exists an algorithm which, given regular (resp. monotone) ETA $\mathcal{A}_{1} / \mathcal{E}, \ldots, \mathcal{A}_{n} / \mathcal{E}$, computes a regular (resp. monotone) ETA $\mathcal{A} / \mathcal{E}$ such that $\mathcal{L}(\mathcal{A} / \mathcal{E})=\operatorname{op}\left(\mathcal{L}\left(\mathcal{A}_{1} / \mathcal{E}\right), \ldots, \mathcal{L}\left(\mathcal{A}_{n} / \mathcal{E}\right)\right)$. One should note that non-regular and equational tree automata defined in [23] are in the above monotone case. It is folklore that whenever $\mathcal{E}=\varnothing$ then $\varnothing$-regular and $\varnothing$ monotone languages coincide. Things are different when some equational theory is taken into account. For instance, it has been shown in [24] that monotone A-TA are strictly more expressive than regular A-TA. But the question remained open in the case of AC.

We sum up in the table of Fig. 1 some known results concerning respectively regular AC-TA, monotone A-TA and monotone AC-TA. The positive results are marked with "Yes", and the negative cases are marked with "No". In case the results are proved in our previous work, the references are omitted. The complexity of the emptiness for regular AC-TA is a direct consequence of Lemma 2 in [23] and the result of regular TA [5]. Question marks "?" in the three columns denote open problems registered in [6].

For most of the results described in the present paper, we will consider a rather simple signature consisting of finitely many constant symbols and a single AC symbol $f$. In this case, the regular transition rules $\mathrm{f}\left(p_{1}, p_{2}\right) \rightarrow q$ and a $\rightarrow q$ correspond to the production rules $q \rightarrow p_{1} p_{2}$ and $q \rightarrow$ a of context-free grammars in Chomsky normal form. In case of monotone TA, the additional form $\mathrm{f}\left(p_{1}, p_{2}\right) \rightarrow \mathrm{f}\left(q_{1}, q_{2}\right)$ together with the previous two forms corresponds to context-sensitive grammar in Kuroda normal form [20]. Following the same approach for monotone AC-TA, the transition rules correspond to the production rules of some commutative context-sensitive grammar. The commutative context-sensitive grammars are known to be close to Petri nets [10]. Therefore, most of our developments are related to Petri nets. For this reason, on the other hand, the com- 
plexity of the emptiness problem for monotone AC-TA is unclear, that may correspond to the reachability problem for Petri nets [8].

\section{The Complexity of the Membership Problem}

In this section, we investigate the complexity of the membership problem for monotone AC-tree automata. To show in particular the PSPACE-hardness, we use a proof technique proposed by Esparza [9] where he shows that the reachability problem for one-safe Petri nets is PSPACE-hard. Note that Petri nets corresponding to monotone AC-tree automata are not in general one-safe.

Theorem 1. Given a monotone AC-tree automaton $\mathcal{A} / \mathrm{AC}$ and a term $t$, the problem whether $t \in \mathcal{L}(\mathcal{A} / \mathrm{AC})$ is PSPACE-complete.

To show that the membership problem for monotone AC-TA is in PSPACE, it suffices to prove that the size of any ground term $t$ reachable from an initial term $t_{0}$ by the move relation of $\mathcal{A} / \mathrm{AC}$ is polynomial relative to the size of $t_{0}$ and $\mathcal{A} / \mathrm{AC}$. This allows us to prove that the existence of a successful run for $t_{0}$ implies that there exists a "short" successful run at most exponential with respect to the size of $t_{0}$ and $\mathcal{A} / \mathrm{AC}$. We use this property to devise a non-deterministic polynomial space algorithm for the membership problem using that the execution of the move relation can be done in polynomial time. We appeal to Savitch's theorem [28] stating that NPSPACE = PSPACE to conclude.

Let us define the special notation of terms. We assume that a term $t$ in this section is represented by the following grammar:

$$
t::=f\left\langle t_{1}, \ldots, t_{n}\right\rangle \mid a
$$

where $f$ is a function symbol in $\mathcal{F}$ with $\operatorname{arity}(f)>0$, and $a$ is a constant. Moreover, $\left\langle t_{1}, \ldots, t_{n}\right\rangle$ is a non-empty sequence of terms $t_{1}, \ldots, t_{n}$ such that:

1. if $f$ is a non-AC symbol, then $n$ is the arity of $f$,

2. if $f$ is an AC symbol, then $n \geqslant 2$ and the root symbol of $t_{i}$ is not $f$.

Given a subterm position and a rule to be applied at the subterm position, the corresponding transition step by $\mathcal{A} / \mathrm{AC}$ can be performed on the above term representation in linear time with respect to the size of a term. In the transition steps, there are two non-standard cases, that are done by the transitions rules of the form $f\left(p_{1}, p_{2}\right) \rightarrow q$ and $f\left(p_{1}, p_{2}\right) \rightarrow f\left(q_{1}, q_{2}\right)$ with $f$ an AC symbol. In both of the two cases, instead of the standard pattern matching, we find $p_{1}, p_{2}$ among subterms $t_{1}, \ldots, t_{n}$ of $f\left\langle t_{1}, \ldots, t_{n}\right\rangle$. By definition of monotone AC-TA, if a term $s$ is reachable from $t$ by $\rightarrow_{\mathcal{A} / \mathrm{AC}}$, the size $|s|$ is less than or equal to $|t| * \log (|\mathcal{A} / \mathrm{AC}|)$, where $|\mathcal{A} / \mathrm{AC}|$ is the number of state symbols of $\mathcal{A} / \mathrm{AC}$. Then we can show that for any tree $t$ admitting a successful run $r: t \rightarrow_{\mathcal{A} / \mathrm{AC}}^{*} q$ with $q$ a final state of $\mathcal{A} / \mathrm{AC}$, there exists a successful run $r^{\prime}: t \rightarrow_{\mathcal{A} / \mathrm{AC}}^{*} q$ reaching the same state $q$ of the length at most $2^{|t| * \log (|\mathcal{A} / \mathrm{AC}|)}$. In fact, for the proof by contradiction, we suppose that $t \rightarrow{ }_{\mathcal{A} / \mathrm{AC}}^{*} q$ is the shortest successful run whose length is strictly greater than $2^{|t| * \log (|\mathcal{A} / \mathrm{AC}|)}$. Then terms reachable from $t$ by $\rightarrow \mathcal{A} / \mathrm{AC}$ can be described using a space relative to the size at most $|t| * \log (|\mathcal{A} / \mathrm{AC}|)$. This implies that the previous shortest run $t \rightarrow_{\mathcal{A} / \mathrm{AC}}^{*} q$ can be represented as $t \rightarrow_{\mathcal{A} / \mathrm{AC}}^{*} u \rightarrow_{\mathcal{A} / \mathrm{AC}}^{+} u \rightarrow_{\mathcal{A} / \mathrm{AC}}^{*} q$. By 
shrinking this run by chopping off the loop of $u$, one can obtain a successful run strictly shorter than the original, leading to the contradiction with respect to the minimality assumption.

Based on the above observation, let us define (non-deterministic) algorithm to solve the question if $t \in \mathcal{L}(\mathcal{A} / \mathrm{AC})$. We write in the algorithm apply $\left(u, u^{\prime}, r\right)$ for denoting to "apply the transition rule $r$ at the position of a subterm $u^{\prime}$ of $u$." This algorithm needs for the computation a polynomially bounded space with respect to the size $|t|+$ $|\mathcal{A} / \mathrm{AC}|$ : Let $t$ be a term over the signature $\mathcal{F}$ and $\mathcal{A} / \mathrm{AC}$ a monotone AC-TA with $\mathcal{A}=\left(\mathcal{F}, \mathcal{Q}, \mathcal{Q}_{\text {fin }}, \Delta\right)$.

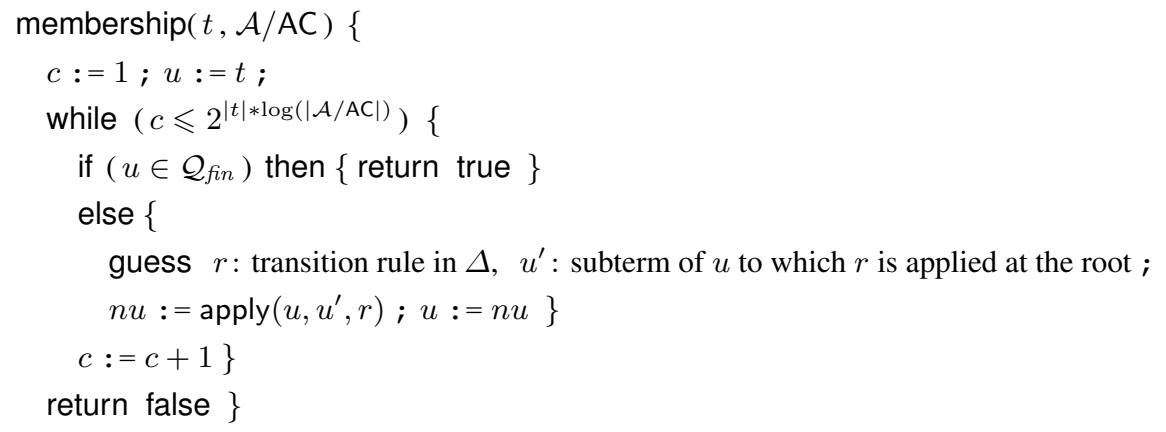

Let us estimate the space complexity of this algorithm. One can see that apply runs in polynomial time, and thus, in polynomial space. For membership we observe that this procedure requires the space for the counter $c$ and the terms $u, u^{\prime}$ and $n u$. Obviously this space can be bounded linearly in $|t| * \log (|\mathcal{A} / \mathrm{AC}|)$. So, membership can be executed by a non-deterministic machine using polynomial space.

Next, to show that the membership problem is PSPACE-hard, we consider the validity problem for closed quantified Boolean formulas (QBF). This problem is known to be PSPACE-complete. Every formula $\varphi$ can be represented by the following grammar:

$$
\varphi::=x|\neg \varphi| \varphi \wedge \varphi \mid \exists x . \varphi \quad(x: \text { a proposition variable) }
$$

This assumption is justified by the fact that any quantified Boolean formula can be translated into a formula of the above form in linear time. We assume also that each variable $x$ in the formula occurs in the scope of some quantifier $\exists x$ or $\forall x$ and that each variable is bounded exactly once in the formula.

We suppose that $x_{1}, \ldots, x_{k}$ are variables bounded in $\varphi$. We show in the following that we can build from a closed formula $\varphi$ a monotone AC-tree automaton $\mathcal{A}_{\varphi} / \mathrm{AC}$ and a term $t_{\varphi}$ in polynomial time relative to the size of $\varphi$ such that $t_{\varphi}$ is accepted by $\mathcal{A}_{\varphi} / \mathrm{AC}$ if and only if $\varphi$ is valid. For this construction, we take the signature $\{\oplus, \mathrm{i}, \mathrm{v}, \mathrm{e}\}$, where $\oplus$ is an $\mathrm{AC}$ symbol and $\mathrm{i}, \mathrm{v}, \mathrm{e}$ are constants. We denote by $t_{\varphi}$ a term consisting of exactly $k$ constants of $\mathrm{v}$, a constant of $\mathrm{i}$, and a constant of e. For each subformula $\psi$ of $\varphi$, we define the state symbols $q_{(\psi, ?)}, q_{(\psi, T)}$, and $q_{(\psi, F)}$. In case of $\psi \equiv \exists x . x \wedge x$, the two subformulas $x$ 's are distinguished in this construction. For each variable $x_{i}$ $(1 \leqslant i \leqslant k)$, we take the two states $q_{\text {true } / x_{i}}$ and $q_{\mathrm{false} / x_{i}}$. The state $q_{f i n}$ is the final state. Let us describe the intended meaning of each state symbol. The truth value of the formula $\varphi$ is computed recursively in our encoding. Along this idea, the state $q_{(\psi, ?)}$ means that the subformula $\psi$ can be taken into consideration. When the computation for 
$\psi$ is performed, the state $q_{(\psi, ?)}$ is "transformed" to either $q_{(\psi, T)}$ or $q_{(\psi, F)}$, depending on the truth value of $\psi$. The state $q_{(\psi, T)}$ means that $\psi$ is true, and $q_{(\psi, F)}$ means that $\psi$ is false. The two states $q_{\mathrm{true} / x_{i}}$ and $q_{\mathrm{false} / x_{i}}$ are the environment to store the information for the valuation to $x_{i}$.

Using the above state symbols, next we define the transition rules. For the constants $\mathrm{i}, \mathrm{v}, \mathrm{e}$, we take the following transition rules: $\mathrm{i} \rightarrow q_{(\varphi, ?)}, \quad \mathrm{v} \rightarrow q_{\mathrm{v}}, \quad \mathrm{e} \rightarrow q_{\mathrm{e}}$.

The first rule is used to initiate the computation. We define the transition rules for instantiating a variable $x_{i}(1 \leq i \leq k)$ to true or false:

$$
q_{\left(x_{i}, ?\right)} \oplus q_{\text {true } / x_{i}} \rightarrow q_{\left(x_{i}, T\right)} \oplus q_{\text {true } / x_{i}} \quad q_{\left(x_{i}, ?\right)} \oplus q_{\text {false } / x_{i}} \rightarrow q_{\left(x_{i}, F\right)} \oplus q_{\text {false } / x_{i}}
$$

The rules for negation are defined as follows: for a subformula $\neg \psi$ of the formula $\varphi$,

$$
\begin{aligned}
& q_{(\neg \psi, ?)} \oplus q_{\mathrm{e}} \rightarrow q_{(\psi, ?)} \oplus q_{\mathrm{e}} \\
& q_{(\psi, T)} \oplus q_{\mathrm{e}} \rightarrow q_{(\neg \psi, F)} \oplus q_{\mathrm{e}} \quad q_{(\psi, F)} \oplus q_{\mathrm{e}} \rightarrow q_{(\neg \psi, T)} \oplus q_{\mathrm{e}} .
\end{aligned}
$$

The first rule decomposes $\neg \psi$ and the last two rules re-construct $\neg \psi$ with the truth value by using $\psi$ with the truth value. Similarly, the rules for the conjunction can be defined. For any subformula $\psi \wedge \psi^{\prime}$ of the formula $\varphi$,

$$
\begin{array}{lll}
q_{\left(\psi \wedge \psi^{\prime}, ?\right)} \oplus q_{\mathrm{e}} \rightarrow q_{(\psi, ?)} \oplus q_{\mathrm{e}} & \\
q_{(\psi, F)} \oplus q_{\mathrm{e}} \rightarrow q_{\left(\psi \wedge \psi^{\prime}, F\right)} \oplus q_{\mathrm{e}} & q_{(\psi, T)} \oplus q_{\mathrm{e}} \rightarrow q_{\left(\psi^{\prime}, ?\right)} \oplus q_{\mathrm{e}} \\
q_{\left(\psi^{\prime}, F\right)} \oplus q_{\mathrm{e}} \rightarrow q_{\left(\psi \wedge \psi^{\prime}, F\right)} \oplus q_{\mathrm{e}} & q_{\left(\psi^{\prime}, T\right)} \oplus q_{\mathrm{e}} \rightarrow q_{\left(\psi \wedge \psi^{\prime}, T\right)} \oplus q_{\mathrm{e}}
\end{array}
$$

In the above definition, $\psi \wedge \psi^{\prime}$ is evaluated in a sequential manner: first we consider the subformula $\psi$ and evaluate it, and then we take the remaining subformula $\psi^{\prime}$. For the existential quantification $\exists x_{i} . \psi$, we need to consider both valuations for the bound variable $x_{i}$ and the computation for $\psi$ :

$$
\begin{array}{ll}
q_{\left(\exists x_{i} . \psi, ?\right)} \oplus q_{\mathrm{v}} \rightarrow q_{\text {true } / x_{i}} \oplus q_{(\psi, ?)} \\
q_{\text {true } / x_{i}} \oplus q_{(\psi, T)} \rightarrow q_{\left(\exists x_{i} . \psi, T\right)} \oplus q_{\text {true } / x_{i}} & q_{\text {true } / x_{i}} \oplus q_{(\psi, F)} \rightarrow q_{\text {false } / x_{i}} \oplus q_{(\psi, ?)} \\
q_{\text {false } / x_{i}} \oplus q_{(\psi, T)} \rightarrow q_{\left(\exists x_{i} . \psi, T\right)} \oplus q_{\text {false } / x_{i}} & q_{\text {false } / x_{i}} \oplus q_{(\psi, F)} \rightarrow q_{\left(\exists x_{i} . \psi, F\right)} \oplus q_{\text {false } / x_{i}}
\end{array}
$$

In the above definition, we start with the valuation associating the Boolean value true with $x_{i}$. If $\psi$ turns out to be true under this valuation, $\exists x . \psi$ is also true; otherwise, the valuation associating the Boolean value false with $x_{i}$ is tried. The following rules are used to finalize the computation:

$$
\oplus\left(q_{(\varphi, T)}, q_{\mathrm{e}}\right) \rightarrow q_{\text {fin }} \quad \oplus\left(q_{\text {true } / x_{i}}, q_{f}\right) \rightarrow q_{\text {fin }} \quad \oplus\left(q_{\text {false } / x_{i}}, q_{f}\right) \rightarrow q_{\text {fin }}
$$

We can show that the previous encoding is correct, by using the induction over the structure of the formula $\psi$. The remainder of the proof is obtained from the following observation: Let $t_{(\psi, ?)}$ be a term that contains exactly a $q_{(\psi, ?)}$, a $q_{\mathrm{e}}$, and $n_{v}$ occurrences of $c_{v}$ ( $c_{v}$ being either the constant $v$ or the state $q_{\mathrm{v}}$, and $n_{v}$ being the number of variables that do not freely occur in $\psi$ ) and for each free variable $x_{i_{1}}, \ldots, x_{i_{\ell}}$, either $q_{\text {true } / x_{i_{j}}}$ or $q_{\mathrm{false} / x_{i_{j}}}$. Suppose $\delta$ is the Boolean valuation defined for $x_{i_{1}}, \ldots, x_{i_{\ell}}$ such that $\delta$ associates with $x_{i_{j}}$ the value true if $q_{\text {true } / x_{i}}$ appears in $t_{(\psi, ?)}$, and false otherwise. Then we have: 
- $t_{(\psi, ?)} \rightarrow_{\mathcal{A}_{\varphi} / \mathrm{AC}}^{*} t_{(\psi, T)}$ iff $\psi$ is valid under $\delta, t_{(\psi, T)}$ being the same as $t_{(\psi, ?)}$ except

1. $q_{(\psi, ?)}$ in $t_{(\psi, ?)}$ is replaced by $q_{(\psi, T)}$,

2. if $x_{i_{l+1}}, \ldots, x_{i_{l+m}}$ are bound variables in $\psi$, then $m$ occurrences of $\mathrm{v}$ and $q_{\mathrm{v}}$ in $t_{(\psi, ?)}$ are replaced by $q_{b_{1} / x_{i_{\ell+1}}}, \ldots, q_{b_{m} / x_{i_{\ell+m}}}$ with $b_{1}, \ldots, b_{m} \in\{$ true, false $\}$. - $t_{(\psi, ?)} \rightarrow_{\mathcal{A}_{\varphi} / \mathrm{AC}}^{*} t_{(\psi, F)}$ iff $\psi$ is not valid under $\delta, t_{(\psi, F)}$ being the same as $t_{(\psi, ?)}$ except

1. $q_{(\psi, ?)}$ in $t_{(\psi, ?)}$ is replaced by $q_{(\psi, F)}$,

2. if $x_{i_{l+1}}, \ldots, x_{i_{l+m}}$ are bound variables in $\psi$, then $m$ occurrences of $\mathrm{v}$ and $q_{\mathrm{v}}$ in $t_{(\psi, ?)}$ are replaced by $q_{b_{1} / x_{i_{\ell+1}}}, \ldots, q_{b_{m} / x_{i_{\ell+m}}}$ with $b_{1}, \ldots, b_{m} \in\{$ true, false $\}$.

As is suggested by one of the referees, the proof of PSPACE-hardness for the membership problem could have been obtained by reduction from the reachability problem of 1-conservative Petri nets. In this kind of Petri nets, transition does not change the total number of tokens in the net. We recall that the reachability problem is to decide for a Petri net $N$ and two configurations $m, m^{\prime}$ whether $m^{\prime}$ is reachable from $m$ in $N$. The reachability problem for 1-conservative Petri nets is PSPACE-complete, and moreover, this result holds even for nets in which each transition consumes two tokens [18].

Therefore, given a Petri net $N$ in this type, it is encoded in linear time using transitions in (TYPE 2) of a monotone AC-tree automaton. The initial configuration $m$ is encoded as an input term $t_{m}$ of the membership problem. Transition rules in (TYPE 1) of the same automaton verify that $m$ in $N$ reaches the goal $m^{\prime}$, by replacing all constants in $t_{m}$ by corresponding states, and by reducing a term corresponding to $m^{\prime}$ to a final state.

\section{Expressiveness: Regular vs. Monotone AC-Tree Automata}

Obviously, by definition, monotone $\mathrm{AC}$-tree automata are at least as expressive as regular AC-tree automata. We show in this section that monotone AC-tree automata are strictly more expressive than regular AC-tree automata. In other words, we are going to present a monotone $\mathrm{AC}$-tree automaton whose accepted language can not be defined by any regular AC-tree automaton.

To construct such a tree language, we consider in particular the signature $\mathcal{F}_{\oplus}=\{\oplus\} \cup$ $\mathcal{F}_{0}$ consisting of a single AC symbol $\oplus$ and constant symbols $a_{1}, \ldots, a_{n}(n \geqslant 1)$. We then define the Parikh mapping $\pi$ ([27]) associated with the signature $\mathcal{F}_{\oplus}$ as follows. For a term $t$ in $\mathcal{T}\left(\mathcal{F}_{\oplus}\right), \pi(t)$ is a vector $v$ in $\mathbb{N}^{n}$ such that the $i$-th component $v(i)$ is the number of occurrences of $a_{i}$ in $t$. For instance, $\pi\left(\oplus\left(a_{1}, \oplus\left(a_{3}, a_{1}\right)\right)\right)=(2,0,1,0, \ldots, 0)$. The Parikh mapping $\pi$ is homomorphically extended to tree languages: for a tree language $L$ over $\mathcal{F}_{\oplus}, \pi(L)$ is the set of vectors in $\mathbb{N}^{n}$ defined as $\pi(L)=\{\pi(t) \mid t \in L\}$.

Proposition 1 ([4]). Given an AC-regular tree language $L$ over $\mathcal{F}_{\oplus}$, the set $\pi(L)$ is a semi-linear set over $\mathbb{N}^{n}$.

The reverse of the above property also holds; for a semi-linear set $S$, there effectively exists an AC-regular tree language $L$ with $\pi(L)=S$. We recall that a subset $S$ of $\mathbb{N}^{n}$ is called a linear set if $S=\operatorname{Lin}\left(b, p_{1}, \ldots, p_{k}\right)$, where $b$ is a vector, called base, in $\mathbb{N}^{n}$ and $p_{1}, \ldots, p_{k}$ are a finite number $k$ of vectors, called periods, such that

$$
\operatorname{Lin}\left(b, p_{1}, \ldots, p_{k}\right)=\left\{b+\sum_{i=1}^{k}\left(\lambda_{i} \times p_{i}\right) \mid \lambda_{1}, \ldots, \lambda_{k} \in \mathbb{N}\right\}
$$


A finite union of such linear sets is called a semi-linear set.

Lemma 1. Suppose $\mathcal{F}_{\oplus}$ is defined with 5 constants. There exists a monotone AC-tree automaton $\mathcal{A}_{\leqslant} /$AC over $\mathcal{F}_{\oplus}$ defining a tree language $L_{\leqslant}$such that

$$
\pi\left(L_{\leqslant}\right)=\left\{\left(k_{1}, k_{2}, k_{3}, 1,2\right) \mid k_{3} \leqslant k_{1} \times k_{2} \text { for } k_{1}, k_{2}, k_{3} \in \mathbb{N}\right\} .
$$

Proof. We take a,b,c,\#,s for the constants of $\mathcal{F}_{\oplus}$. The corresponding Parikh images are the numbers of these constants in the above order. We define the tree automaton $\mathcal{A}_{\leqslant}=\left(\mathcal{F}_{\oplus}, \mathcal{Q}, \mathcal{Q}_{\text {fin }}, \Delta_{\leqslant}\right)$over $\mathcal{F}_{\oplus}$, where $\mathcal{Q}=\left\{p_{\mathrm{a}}, p_{\mathrm{b}}, p_{\mathrm{c}}, p_{\#}, p_{\mathrm{s}}, p_{\text {fin }}, q_{\mathrm{a}}, q_{\#}, q_{\mathrm{s}}, r_{\#}\right\}$, $\mathcal{Q}_{\text {fin }}=\left\{p_{\text {fin }}\right\}$ and

$$
\begin{aligned}
& \Delta_{\leqslant}: \mathrm{a} \rightarrow p_{\mathrm{a}} \quad \mathrm{b} \rightarrow p_{\mathrm{b}} \quad \mathrm{c} \rightarrow p_{\mathrm{c}} \quad \# \rightarrow p_{\#} \quad \mathrm{~s} \rightarrow q_{\mathrm{s}} \quad q_{\mathrm{s}} \oplus q_{\mathrm{s}} \rightarrow p_{\mathrm{s}} \\
& p_{\#} \oplus p_{\mathrm{a}} \rightarrow p_{\#} \quad p_{\#} \oplus p_{\mathrm{a}} \rightarrow q_{\#} \oplus q_{\mathrm{a}} \\
& q_{\#} \oplus p_{\mathrm{c}} \rightarrow p_{\#} \quad p_{\#} \oplus p_{\mathrm{b}} \rightarrow r_{\#} \\
& r_{\#} \oplus q_{\mathrm{a}} \rightarrow r_{\#} \oplus p_{\mathrm{a}} \quad r_{\#} \oplus p_{\mathrm{s}} \rightarrow p_{\#} \oplus p_{\mathrm{s}} \quad p_{\#} \oplus p_{\mathrm{s}} \rightarrow p_{\text {fin }}
\end{aligned}
$$

We denote by $|t|_{\alpha}$ the number of occurrences of a constant $\alpha\left(\in \mathcal{F}_{0} \cup \mathcal{Q}\right)$ in a term $t$ over $\mathcal{F}_{\oplus} \cup \mathcal{Q}$. We observe that for any term $t$ over $\mathcal{F}_{\oplus}$ such that $|t|_{\#}=1$ and $|t|_{\mathrm{s}}=2$ and $|t|_{\mathrm{c}} \leqslant|t|_{\mathrm{a}} \times|t|_{\mathrm{b}}$, there exists a derivation $t \rightarrow_{\mathcal{A}_{\leqslant} / \mathrm{AC}}^{*} p_{\text {fin }}$ from $t$ to $p_{\text {fin }}$. In order to prove this observation, let us define the assertions and the algorithm in Fig.2. The function apply in the algorithm corresponds to a single application of its argument to a term in consideration. The derivation of $t$ is the sequence of terms obtained during the computation. Proofs of correctness and termination easily follow from the annotations. Conversely, for any term $t_{0}$ over $\mathcal{F}_{\oplus}$ and $t$ over $\mathcal{F}_{\oplus} \cup \mathcal{Q}$, if $t_{0} \rightarrow_{\mathcal{A}_{\leqslant} / \text {AC }}^{*} t$, it holds that:

$$
\begin{aligned}
& \left|t_{0}\right|_{\mathrm{s}}=\left(|t|_{\mathrm{s}}+|t|_{q_{\mathrm{s}}}\right)+2 \times\left(|t|_{p_{\text {fin }}}+|t|_{p_{\mathrm{s}}}\right) \\
& \left|t_{0}\right|_{\#}=|t|_{\#}+|t|_{p_{\#}}+|t|_{q_{\#}}+|t|_{r_{\#}}+|t|_{p_{\text {fin }}} \\
& \left|t_{0}\right|_{\mathrm{a}} \geqslant|t|_{\mathrm{a}}+|t|_{p_{\mathrm{a}}}+|t|_{q_{\mathrm{a}}}
\end{aligned}
$$

Moreover, if $t_{0} \rightarrow_{\mathcal{A}_{\leqslant} / \text {AC }}^{*} p_{\text {fin }}$, then by (INV 1), $\left|t_{0}\right|_{\mathrm{s}}=2$ and by (INV 2), $\left|t_{0}\right|_{\#}=1$. Now we suppose $\left|t_{0}\right|_{\#}=1$. Due to (INV 3 ), we have

$$
\left|t_{0}\right|_{\mathrm{c}}-\left(|t|_{\mathrm{c}}+|t|_{p_{\mathrm{c}}}\right) \leqslant\left|t_{0}\right|_{\mathrm{a}} \times\left(\left|t_{0}\right|_{\mathrm{b}}-\left(|t|_{\mathrm{b}}+|t|_{p_{\mathrm{b}}}\right)\right)+|t|_{q_{\mathrm{a}}} \times\left(1-|t|_{r_{\#}}\right)-|t|_{q_{\#}} .
$$

Accordingly, if $t_{0} \rightarrow_{\mathcal{A}_{\leqslant} / \text {AC }}^{*} p_{\text {fin }}$, then $\left|t_{0}\right|_{\mathrm{c}} \leqslant\left|t_{0}\right|_{\mathrm{a}} \times\left|t_{0}\right|_{\mathrm{b}}$. Therefore, $t_{0} \in L_{\leqslant}$if and only if $\pi\left(t_{0}\right)=\left(k_{1}, k_{2}, k_{3}, 1,2\right)$ with $k_{3} \leqslant k_{1} \times k_{2}$.

Theorem 2. The family of AC-regular tree languages is properly included in the family of AC-monotone tree languages.

Proof. Straightforward from Proposition 1 and Lemma 1, because the Parikh image of $L_{\leqslant}$is not semi-linear. 
$/^{*}$ Given $t$ in $\mathcal{T}\left(\mathcal{F}_{\oplus}\right)$ such that $|t|_{\#}=1$ and $|t|_{\mathrm{s}}=2$ and $|t|_{\mathrm{c}} \leqslant|t|_{\mathrm{a}} \times|t|_{\mathrm{b}} \quad$ */

while $\left(|t|_{\mathrm{a}}+|t|_{\mathrm{b}}+|t|_{\mathrm{c}}+|t|_{\#}+|t|_{\mathrm{s}}>0\right)\{$

apply $\left.\mathrm{a} \rightarrow p_{\mathrm{a}}, \mathrm{b} \rightarrow p_{\mathrm{b}}, \mathrm{c} \rightarrow p_{\mathrm{c}}, \# \rightarrow p_{\#}, \mathbf{s} \rightarrow q_{\mathrm{s}}\right\}$

$/{ }^{*}$ INVARIANT :

$$
\begin{aligned}
& |t|_{p_{\mathrm{c}}}+|t|_{q_{\mathrm{a}}}+\left(|t|_{p_{\mathrm{a}}} \times|t|_{r_{\#}}\right) \leqslant\left(|t|_{p_{\mathrm{a}}}+|t|_{q_{\mathrm{a}}}\right) \times\left(|t|_{p_{\mathrm{b}}}+|t|_{r_{\#}}\right)+|t|_{q_{\#}} \\
& |t|_{p_{\#}}+|t|_{q_{\#}}+|t|_{r_{\#}}=|t|_{p_{\mathrm{s}}}=1 \\
& |t|_{\mathrm{a}}+|t|_{\mathrm{b}}+|t|_{\mathrm{c}}=0
\end{aligned}
$$

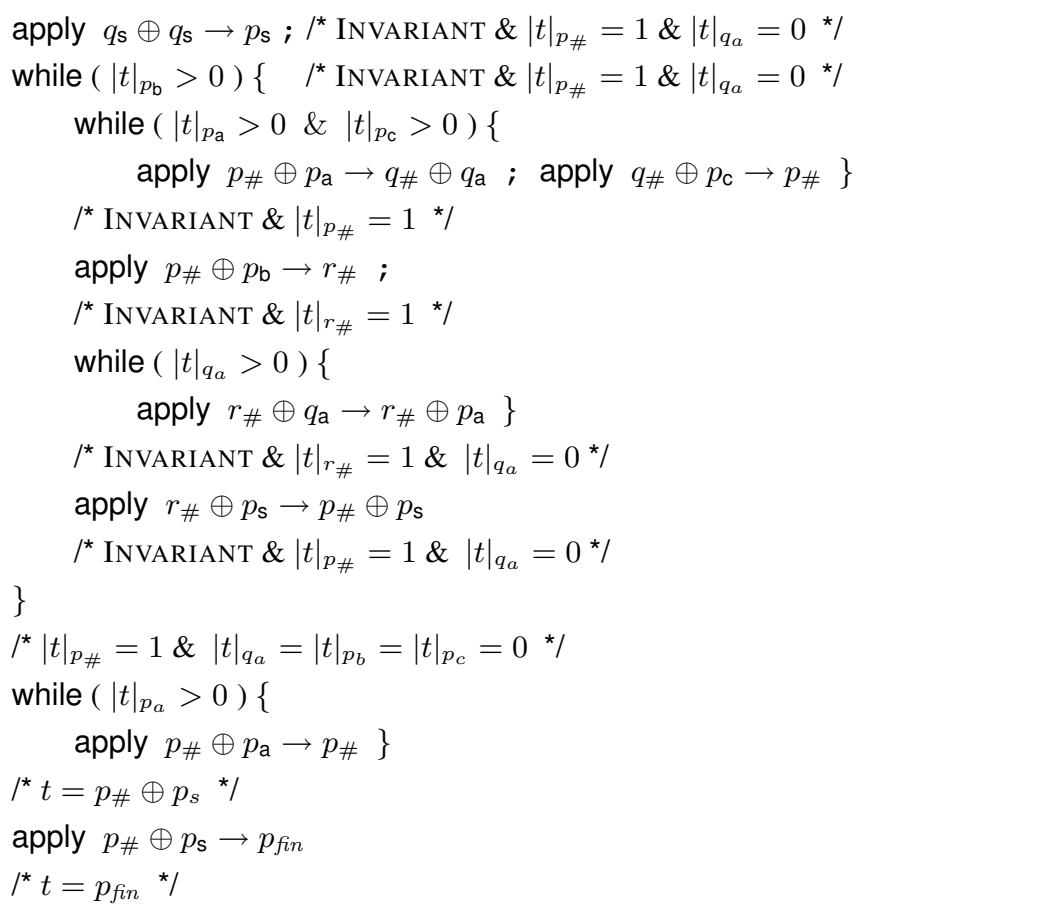

Fig. 2. Reduction strategy and the assertions

\section{Complementation of AC-Monotone Tree Languages}

As is explained in the introduction, monotone rules in tree case correspond to contextsensitive grammars in word case. In fact, based on this observation, we proved in a previous paper [24] that A-monotone tree languages are closed under Boolean operations by reduction from the fact that context-sensitive languages are closed under complementation. In this section, however, we show that AC-monotone tree languages are not closed under complementation. 
Theorem 3. There exists an AC-monotone tree language whose complement is not an AC-monotone tree language.

In the remaining part of this section, we devote to show the proof of Theorem 3. Our proof proceeds in the way of proof by contradiction.

Lemma 2. Suppose $\mathcal{F}_{\oplus}$ is defined with 5 constants. There exists an AC-tree automaton $\mathcal{A}_{<} /$AC over $\mathcal{F}_{\oplus}$ defining a tree language $L_{<}$such that

$$
L_{<}=\left\{\left(k_{1}, k_{2}, k_{3}, 1,2\right) \mid k_{3}<k_{1} \times k_{2} \text { for } k_{1}, k_{2}, k_{3} \in \mathbb{N}\right\} .
$$

Proof. We define the automaton $\mathcal{A}_{<} / \mathrm{AC}$ exactly as is the monotone $\mathrm{AC}$-tree automaton $\mathcal{A}_{\leqslant} / \mathrm{AC}$ in Lemma 1 except that the rule $q_{\mathrm{s}} \oplus q_{\mathrm{s}} \rightarrow p_{\mathrm{s}}$ is replaced by the rule $q_{\mathrm{s}} \oplus q_{\mathrm{s}} \rightarrow$ $p_{\mathrm{s}} \oplus p_{\mathrm{c}}$. One can show as we have done for $\mathcal{A}_{\leq} / \mathrm{AC}$, that for any term $t_{0}$ in $\mathcal{T}\left(\mathcal{F}_{\oplus}\right)$, $t_{0} \rightarrow_{\mathcal{A}_{<} / \mathrm{AC}}^{*} p_{\text {fin }}$ if and only if $\pi\left(t_{0}\right)=\left(k_{1}, k_{2}, k_{3}, 1,2\right)$ with $k_{3}<k_{1} \times k_{2}$.

Let us consider the tree language $L \geqslant$ defined below over the above signature $\mathcal{F}_{\oplus}=$ $\{\oplus\} \cup\{\mathrm{a}, \mathrm{b}, \mathrm{c}, \#, \mathrm{~s}\}:$

$$
L \geqslant=\left\{\left(k_{1}, k_{2}, k_{3}, 1,2\right) \mid k_{3} \geqslant k_{1} \times k_{2} \text { for } k_{1}, k_{2}, k_{3} \in \mathbb{N}\right\},
$$

and we take the hypothesis

$\mathcal{H}: L \geqslant$ is an AC-monotone tree language.

We then state the following property associated to $\mathcal{H}$.

Lemma 3. If $\mathcal{H}$ holds, there exists a monotone AC-tree automaton that accepts $L_{=}$ over $\mathcal{F}_{\oplus}$ such that $\pi\left(L_{=}\right)=\left\{\left(k_{1}, k_{2}, k_{3}, 1,2\right) \mid k_{3}=k_{1} \times k_{2}\right.$ for $\left.k_{1}, k_{2}, k_{3} \in \mathbb{N}\right\}$.

Proof. Due to $\mathcal{H}$, there exists a monotone $\mathrm{AC}$-tree automaton $\mathcal{A} \geqslant / \mathrm{AC}$ with $\mathcal{L}(\mathcal{A} \geqslant / \mathrm{AC})=$ $L \geqslant$. It is known that the class of monotone AC-tree automata is effectively closed under intersection (Theorem 3, [23]). Then we let $\mathcal{B} / \mathrm{AC}$ be the intersection of $\mathcal{A}_{\leqslant} / \mathrm{AC}$ in the previous section and $\mathcal{A} \geqslant / A C$. According to the trivial fact that $\left(n_{1} \geqslant n_{2}\right) \wedge\left(n_{1} \leqslant n_{2}\right)$ if and only if $n_{1}=n_{2}, \mathcal{B} / \mathrm{AC}$ accepts $L_{\geqslant} \cap L_{\leqslant}$, and therefore, $\mathcal{B} / \mathrm{AC}$ accepts $L_{=}$.

Lemma 4. If $\mathcal{H}$ holds, there exists an algorithm that takes as an input $\mathcal{D}$ a diophantine equation and returns as an output "yes" if $\mathcal{D}$ admits a non-negative solution; otherwise, "no".

Proof. Let us assume a finite set of variables $x_{1}, \ldots, x_{n}$ ranging over the natural numbers $\mathbb{N}$. We consider a system of numerical equations $\mathcal{S}=\left\{\mathrm{Eq}_{1}, \ldots, \mathrm{Eq}_{m}\right\}$, where each $\mathrm{Eq}_{\ell}(1 \leqslant \ell \leqslant m)$ in $\mathcal{S}$ is in one of the following forms:

$$
x_{i}=c \quad(c: \text { a fixed natural number }) \quad x_{i}=x_{j}+x_{k} \quad x_{i}=x_{j} \times x_{k}
$$

Here $i$ must be different from $j$ and $k$, i.e. $x_{i}$ does not occur in the right-hand side of the same equation. But a variable $x_{i}$ may occur in the left hand-sides of different equations. A solution $\sigma$ for an equation $\mathrm{Eq}_{\ell}$ is a mapping from $\left\{x_{1}, \ldots, x_{n}\right\}$ to $\mathbb{N}$, such that the structure $(\mathbb{N},+, *,=)$ is a model of $\mathrm{Eq}_{\ell}$ under the valuation $\sigma$. A solution $\sigma$ for a system $\mathcal{S}$ is a solution for every equation in $\mathcal{S}$. 
It is well-known that from any diophantine equation $\mathcal{D}$, one can compute a system of numerical equations $\mathcal{S}$ such that $\mathcal{D}$ admits a solution if and only if $\mathcal{S}$ admits a solution. Now, for each equation $\mathrm{Eq}_{\ell}$ in $\mathcal{S}$, we define a monotone AC-TA $\mathcal{A}_{\mathrm{Eq}_{\ell} / \mathrm{AC}}$ over the signature $\mathcal{F}_{\oplus}=\{\oplus\} \cup\left\{\mathrm{a}_{1}, \ldots, \mathrm{a}_{n}, \#, \mathbf{s}\right\}$, such that for any term $t$ in $\mathcal{T}\left(\mathcal{F}_{\oplus}\right), t \in$ $\mathcal{L}\left(\mathcal{A}_{\mathrm{Eq}_{\ell}} / \mathrm{AC}\right)$ if and only if $|t|_{\#}=1,|t|_{s}=2$ and the valuation $\sigma$ defined as $\sigma\left(x_{i}\right)=$ $|t|_{\mathrm{a}_{i}}$ (for $1 \leqslant i \leqslant n$ ) is a solution for $\mathrm{Eq}_{\ell}$. For each kind of numerical equations, we define the transition rules of the automaton assuming that $p_{f i n}$ is the unique final state:

- For the constraint equation $x_{i}=0$ we define the tree automaton $\mathcal{A}_{x_{i}=0}$ equipped with the transition rules

$$
\begin{aligned}
& \left\{p_{\mathrm{s}} \oplus p_{\mathrm{s}} \rightarrow q_{\mathrm{s}}, q_{\mathrm{s}} \oplus p_{\#} \rightarrow p_{\text {fin }}\right\} \cup \\
& \left\{p_{\mathrm{a}_{j}} \oplus p_{\text {fin }} \rightarrow p_{\text {fin }} \mid j \neq i \text { and } 1 \leqslant j \leqslant n\right\}
\end{aligned}
$$

with the rules for constants $\left\{\mathbf{a}_{j} \rightarrow p_{\mathbf{a}_{j}} \mid 1 \leqslant j \leqslant n\right\} \cup\left\{\# \rightarrow p_{\#}, \mathbf{s} \rightarrow p_{\mathbf{s}}\right\}$. For $x_{i}=c(c>0)$ we additionally take the transition rules

$$
\begin{aligned}
& \left\{p_{\mathbf{a}_{i}} \oplus p_{\text {fin }} \rightarrow p_{1}\right\} \cup\left\{p_{\mathbf{a}_{i}} \oplus p_{j} \rightarrow p_{j+1} \mid 1 \leqslant j \leqslant c-2\right\} \cup \\
& \left\{p_{\mathbf{a}_{i}} \oplus p_{c-1} \rightarrow p_{\text {fin }}\right\} .
\end{aligned}
$$

- For the linear equation $x_{i}+x_{j}=x_{k}$ we define the tree automaton $\mathcal{A}_{x_{i}+x_{j}=x_{k}}$ equipped with the transition rules

$$
\begin{aligned}
& \left\{p_{\mathrm{s}} \oplus p_{\mathrm{s}} \rightarrow q_{\mathrm{s}}, q_{\mathrm{s}} \oplus p_{\#} \rightarrow p_{\text {fin }}\right\} \cup\left\{p_{\mathrm{a}_{i}} \oplus p_{\mathrm{a}_{k}} \rightarrow p, p_{\mathrm{a}_{j}} \oplus p_{\mathrm{a}_{k}} \rightarrow p\right\} \cup \\
& \left\{p_{\mathrm{a}_{\ell}} \oplus p_{\text {fin }} \rightarrow p_{\text {fin }} \mid \ell \neq i \text { and } \ell \neq j \text { and } \ell \neq k\right\} \cup \\
& \left\{p \oplus p \rightarrow p, p \oplus p_{\text {fin }} \rightarrow p_{\text {fin }}\right\}
\end{aligned}
$$

with the rules for constants $\left\{\mathbf{a}_{\ell} \rightarrow p_{\mathbf{a}_{\ell}} \mid 1 \leqslant \ell \leqslant n\right\} \cup\left\{\# \rightarrow p_{\#}, \mathbf{s} \rightarrow p_{\mathbf{s}}\right\}$.

- Finally, for a numerical equation $x_{i}=x_{j} \times x_{k}$, we build the automaton $\mathcal{A}_{x_{i}=x_{j} \times x_{k}}$; let $\mathcal{B} / A C$ the automaton defined in the proof of Lemma 3. We assume without loss of generality that $p_{\text {fin }}$ is the unique final state of $\mathcal{B} / \mathrm{AC}$. We then define $\mathcal{A}_{x_{i}=x_{j} \times x_{k}}$ by relabeling $\mathrm{c}$ by $\mathrm{a}_{i}$, a by $\mathrm{a}_{j}$ and $\mathrm{b}$ by $\mathrm{a}_{k}$ and by adding the transition rules

$$
\begin{aligned}
& \left\{p_{\mathbf{a}_{\ell}} \oplus p_{\text {fin }} \rightarrow p_{\text {fin }} \mid \ell \neq i \text { and } \ell \neq j \text { and } \ell \neq k\right\} \cup \\
& \left\{\mathbf{a}_{\ell} \rightarrow p_{\mathbf{a}_{\ell}} \mid 1 \leqslant \ell \leqslant n\right\} .
\end{aligned}
$$

One should note that for the first two cases, transition rules for \# and $\mathrm{s}$ are not essential, but they must be included under our construction if a system $\mathcal{S}$ contains an equation $\mathrm{Eq}_{k}$ of the multiplication $x_{i}=x_{j} \times x_{k}$.

Accordingly, for the system $\mathcal{S}=\left\{\mathrm{Eq}_{1}, \ldots, \mathrm{Eq}_{m}\right\}$ of numerical equations, we can construct a monotone AC-TA $\mathcal{A}_{\mathcal{S}} / \mathrm{AC}$ such that

$$
\mathcal{L}\left(\mathcal{A}_{\mathcal{S}} / \mathrm{AC}\right)=\bigcap_{1 \leqslant \ell \leqslant m} \mathcal{L}\left(\mathcal{A}_{\mathrm{Eq}_{\ell}} / \mathrm{AC}\right)
$$

whose accepted language is non-empty if and only if $\mathcal{S}$ admits a solution. Since the emptiness problem for monotone AC-TA is decidable, there exists an algorithm under the hypothesis $\mathcal{H}$ that takes as an input a diophantine equation $\mathcal{D}$ and returns "yes" if there is a non-negative solution; otherwise, "no".

It is well-known that Hilbert's 10th problem is undecidable [22], even only in the case of non-negative solutions to be considered. Thus we obtain the following property. 
Theorem 4. There is no monotone AC-tree automaton that accepts $L \geqslant$ over the signature $\mathcal{F}_{\oplus}$.

Corollary 1. The class of AC-monotone tree languages is not closed under complementation.

Proof. Straightforward from Theorem 4, as AC-monotone tree languages are closed under intersection and $L_{\geqslant}=\left(L_{<}\right)^{\mathrm{c}} \cap\left\{\left.t \in \mathcal{T}\left(\mathcal{F}_{\oplus}\right)|| t\right|_{\#}=1 \&|t|_{\mathrm{s}}=2\right\}$, where $L_{<}$ and $\left\{\left.t \in \mathcal{T}\left(\mathcal{F}_{\oplus}\right)|| t\right|_{\#}=1 \&|t|_{\mathrm{s}}=2\right\}$ are AC-monotone tree languages.

\section{The Inclusion Problem for Monotone AC-Tree Automata}

Using the previous tree automata construction, we show in this section that the inclusion problem for AC-monotone tree languages is undecidable. The remainder of this section is devoted to the proof of the following undecidability result.

Theorem 5. Given two monotone $A C$-tree automata $\mathcal{A}_{1} / \mathrm{AC}$ and $\mathcal{A}_{2} / \mathrm{AC}$ over the same signature, the problem whether $\mathcal{L}\left(\mathcal{A}_{1} / \mathrm{AC}\right) \subseteq \mathcal{L}\left(\mathcal{A}_{2} / \mathrm{AC}\right)$ is not decidable.

As we did in the previous section, we consider a system $\mathcal{S}=\left\{\mathrm{Eq}_{1}, \ldots, \mathrm{Eq}_{m}\right\}$ of numerical equations defined over a finite set of variables $\left\{x_{1}, \ldots, x_{n}\right\}$. One should note that according to the syntax, $\mathrm{Eq}_{i}$ is an equation in the form of $x_{j}=e$, where $e$ is either a fixed natural number $c$, the addition $x_{k}+x_{\ell}$, or the multiplication $x_{k} \times x_{\ell}$, such that $x_{j} \neq x_{k}$ and $x_{j} \neq x_{\ell}$.

We then define the system $\mathcal{S}_{\leqslant}$of inequations obtained by replacing each equation $x_{i}=$ $e$ by the inequation $x_{i} \leqslant e$. Namely, $\mathcal{S}_{\leqslant}=\left\{x_{i} \leqslant e \mid x_{i}=e \in \mathcal{S}\right\}$.

Finally we define, for each $k$ with $1 \leqslant k \leqslant m, \mathcal{S}_{k}$ a system of inequations obtained from $\mathcal{S}_{\leqslant}$by replacing only the $k$-th inequation $x_{i} \leqslant e_{k}$ by the strict inequation $x_{i}<e_{k}$. From previous sections, we know that one can effectively associate with each inequation Ineq $_{k}$ (being either $x_{j} \leqslant e_{k}$ or $x_{j}<e_{k}$ ) a monotone AC-tree automaton $\mathcal{A}_{\text {Ineq }_{k}}$ such that a term $t$ from $\mathcal{T}\left(\mathcal{F}_{\oplus}\right)$ is accepted by an automaton $\mathcal{A}_{\text {lneq }_{k}} / \mathrm{AC}$ if and only if $|t|_{\#}=$ $1,|t|_{\mathbf{S}}=2$ and either $\operatorname{lneq}_{k}$ is of the form

$-x_{i} \leqslant c$ and $|t|_{\mathbf{a}_{i}} \leqslant c$ (resp. $x_{i}<c$ and $|t| \mathrm{a}_{i}<c$ ),

$-x_{i} \leqslant x_{v}+x_{w}$ and $|t| \mathbf{a}_{i} \leqslant|t|_{\mathbf{a}_{v}}+|t|_{\mathbf{a}_{w}}$ (resp. $x_{i}<x_{v}+x_{w}$ and $|t|_{\mathbf{a}_{i}}<|t|_{\mathbf{a}_{v}}+$ $\left.|t|_{\mathbf{a}_{w}}\right)$, or

- $x_{i} \leqslant x_{v} * x_{w}$ and $|t| \mathbf{a}_{i} \leqslant|t| \mathbf{a}_{v} *|t| \mathbf{a}_{w}$ (resp. $x_{i}<x_{v} * x_{w}$ and $|t| \mathbf{a}_{i}<|t| \mathbf{a}_{v} *|t| \mathbf{a}_{w}$ ).

Moreover, we let for all $1 \leqslant k \leqslant m$,

$$
\mathcal{A}_{\mathcal{S}_{\leqslant}} / \mathrm{AC}=\bigcap_{\text {Ineq } \in \mathcal{S}_{\leqslant}} \mathcal{A}_{\text {lneq }} / \mathrm{AC}, \quad \mathcal{A}_{\mathcal{S}_{k}} / \mathrm{AC}=\bigcap_{\text {Ineq } \in \mathcal{S}_{k}} \mathcal{A}_{\text {lneq }} / \mathrm{AC}
$$

In the above definition, $\bigcap_{\text {Ineq } \in \mathcal{S}_{\leqslant}} \mathcal{A}_{\text {Ineq }} / \mathrm{AC}$ represents an $\mathrm{AC}$-TA that accepts the tree language accepted by $\mathcal{A}_{\text {lneq }} / \mathrm{AC}$ for all Ineq $\in \mathcal{S}$.

Lemma 5. $\mathcal{L}\left(\mathcal{A}_{\mathcal{S}_{\leqslant}} / \mathrm{AC}\right) \nsubseteq \mathcal{L}\left(\bigcup_{1 \leqslant i \leqslant m} \mathcal{A}_{\mathcal{S}_{i}} / \mathrm{AC}\right)$ if and only if $\mathcal{S}$ admits a solution.

Theorem 5 follows easily from the above Lemma 5 together with the effective closedness under union and intersection of monotone AC-tree automata and the undecidability of Hilbert's 10th problem [22]. 


\section{Concluding Remarks}

In this paper, we have shown the 4 new results (Theorems 1,2, 5 and Corollary 1) for the class of monotone AC-tree automata. Our proof technique used for showing the expressiveness of AC-monotone tree languages explains also a new idea of how to interpret by AC-tree automata the arithmetic constraints over the natural numbers, while an observation obtained from this tree automata construction gives rise to the negative closure property of the complementation and the undecidability of the inclusion problem.

For further research along monotone AC-tree automata, it might be interesting to consider the question about decision problems concerning regularity, called the regularity problem; it is not clear how to determine, given a monotone AC-tree automaton, whether the accepted tree language can also be accepted by some regular AC-tree automaton. Useful ideas to solve this decision problem are found in the study about Petri nets. In fact, it is known that the semi-linearity problem for Petri nets is decidable [15]. The regularity problem for AC-monotone tree languages can be regarded in some sense as an generalization of the above semi-linearity problem.

Another interesting question about monotone AC-tree automata is the universality problem [6]; this problem is known to be decidable for regular AC-tree automata and it is undecidable for monotone A-tree automata.

Acknowledgments. The authors thank anonymous referees for their detailed comments and suggestions to improve the early version of the draft.

\section{References}

1. A. Armando, D. Basin, M. Bouallagui, Y. Chevalier, L. Compagna, S. Mödersheim, M. Rusinowitch, M. Turuani, L. Viganò, and L. Vigneron. The AVISS Security Protocol Analysis Tool. In Proc. of 14th CAV, Copenhagen (Denmark), volume 2404 of LNCS, pages 349-353. Springer, 2002.

2. I. Boneva and J.-M. Talbot. Automata and Logics for Unranked and Unordered Trees. In Proc. of 16th RTA, Nara (Japan), volume 3467 of LNCS, pages 500-515. Springer, 2005.

3. A. Bouhoula, J.P. Jouannaud, and J. Meseguer. Specification and Proof in Membership Equational Logic. TCS, 236:35-132, 2000.

4. T. Colcombet. Rewriting in the Partial Algebra of Typed Terms Modulo AC. In Proc. of 4th INFINITY, Brno (Czech Republic), volume 68(6) of ENTCS. Elsevier, 2002.

5. H. Comon, M. Dauchet, R. Gilleron, F. Jacquemard, D. Lugiez, S. Tison, and M. Tommasi. Tree Automata Techniques and Applications, 2002. (http://www.grappa.univ-lille3.fr/tata).

6. N. Dershowitz and R. Treinen. Problem \#101. The RTA List of Open Problems. Available at http://www.lsv.ens-cachan.fr/rtaloop/ .

7. P. Devienne, J.-M. Talbot, and S. Tison. Set-Based Analysis for Logic Programming and Tree Automata. In Proc. of 4th SAS, Paris (France), volume 1302 of LNCS, pages 127-140. Springer, 1997.

8. J. Esparza. Decidability and Complexity of Petri Net Problems - An Introduction. In Petri Nets, Dagstuhl (Germany), volume 1491 of LNCS, pages 374-428. Springer, 1996.

9. J. Esparza. Decidability of Model-Checking for Infinite-State Concurrent Systems. Acta Informatica, 34:85-107, 1997.

10. J. Esparza. Grammars as Processes. In Formal and Natural Computing - Essays Dedicated to Grzegorz Rozenberg, volume 2300 of LNCS, pages 277-297. Springer, 2002. 
11. J.P. Gallagher and G. Puebla. Abstract Interpretation over Non-Deterministic Finite Tree Automata for Set-Based Analysis of Logic Programs. In Proc. of 4th PADL, Portland (USA), volume 2257 of $L N C S$, pages 243-261. Springer, 2002.

12. T. Genet and F. Klay. Rewriting for Cryptographic Protocol Verification. In Proc. of 17th $C A D E$, Pittsburgh (USA), volume 1831 of $L N C S$, pages 271-290. Springer, 2000.

13. S. Ginsburg. The Mathematical Theory of Context-Free Languages. McGraw-Hill, 1966.

14. J. Goubault-Larrecq and K. N. Verma. Alternating Two-way AC-Tree Automata. Technical Report LSV-02-11, Laboratoire Spécification et Vérification, 2002. (http://www.lsv.enscachan.fr/Publis/).

15. D. Hauschildt. Semilinearity of the Reachability Set is Decidable for Petri Nets. Technical Report FBI-HH-B-146/90, Universität Hamburg, 1990.

16. J. Hendrix, H. Ohsaki, and J. Meseguer. Sufficient Completeness Checking with Propositional Tree Automata. Technical Report AIST-PS-2005-013, National Institute of Advanced Industrial Science and Technology, 2005. (http://staff.aist.go.jp/hitoshi.ohsaki/).

17. H. Hosoya, J. Vouillon, and B. C. Pierce. Regular Expression Types for XML. In Proc. of 5th ICFP, Montreal (Canada), volume 35(9) of SIGPLAN Notices, pages 11-22. ACM, 2000.

18. N. D. Jones, L. H. Landweber, and Y. E. Lien. Complexity of Some Problems in Petri Nets. TCS, 4(3):277-299, 1977.

19. M. Kudlek and V. Mitrana. Normal Forms of Grammars, Finite Automata, Abstract Families, and Closure Properties of Multiset Languages. In Multiset Processing, volume 2235 of LNCS, pages 135-146. Springer, 2001.

20. S. Y. Kuroda. Classes of Languages and Linear Bounded Automata. Information and Control, 7(2):207-223, 1964.

21. D. Lugiez. Counting and Equality Corstraints for Multitree Automata. In Proc. of 6th FOSSACS, Warsaw (Poland), volume 2620 of LNCS, pages 328-342. Springer, 2003.

22. Y. Matiyasevich. Enumerable Sets are Diophantine. Doklady Akademii Nauk SSSR, 191(2):279-282, 1970 (in Russian). Improved and English translation in Soviet Mathematics Doklady, 11:354-357.

23. H. Ohsaki. Beyond Regularity: Equational Tree Automata for Associative and Commutative Theories. In Proc. of 15th CSL, Paris (France), volume 2142 of LNCS, pages 539-553. Springer, 2001.

24. H. Ohsaki, H. Seki, and T. Takai. Recognizing Boolean Closed A-Tree Languages with Membership Conditional Rewriting Mechanism. In Proc. of 14th RTA, Valencia (Spain), volume 2706 of LNCS, pages 483-498. Springer, 2003.

25. H. Ohsaki and T. Takai. Decidability and Closure Properties of Equational Tree Languages. In Proc. of 13th RTA, Copenhagen (Denmark), volume 2378 of LNCS, pages 114-128. Springer, 2002.

26. H. Ohsaki and T. Takai. ACTAS: A System Design for Associative and Commutative Tree Automata Theory. In Proc. of 5th RULE, Aachen (Germany), volume 124(1) of ENTCS, pages 97-111. Elsevier, 2005.

27. R. J. Parikh. On Context-Free Languages. JACM, 13(4):570-581, 1966.

28. W. Savitch. Relationships between Nondeterministic and Deterministic Tape Complexities. Journal of Computer and Systems Sciences, 4(2):177-192, 1970.

29. H. Seidl, T. Schwentick, and A. Muscholl. Numerical Document Queries. In Proc. of 22nd PODS, San Diego (USA), pages 155-166. ACM, 2003.

30. K. N. Verma. On Closure under Complementation of Equational Tree Automata for Theories Extending AC. In Proc. of 10th LPAR, Almaty (Kazakhstan), volume 2850 of LNCS, pages 183-197. Springer, 2003.

31. K. N. Verma. Two-Way Equational Tree Automata for AC-like Theories: Decidability and Closure Properties. In Proc. of 14th RTA, Valencia (Spain), volume 2706 of LNCS, pages 180-197. Springer, 2003. 